\title{
A Framework of Semantic-based Agents to Support Designing in Virtual Environments
}

\author{
Rabee M. Reffat and Kirsty Beilharz \\ Key Centre of Design Computing and Cognition \\ Faculty of Architecture \\ University of Sydney, NSW 2006, Australia \\ Email:rabee@arch.usyd.edu.au \& kirsty@arch.usyd.edu.au
}

\begin{abstract}
In order for a virtual environment to be effective as a designing platform for digital architecture, it is not enough to focus on the precision of the renderings and the accuracy of simulated behaviours. The environment should provide appropriate design support to its users. Design objects are rarely isolated; they are always in the company of other objects. Design spaces are constructed from the relationships between these objects. This paper presents a framework of developing virtual environments in which design semantics (high level relationships between objects) are incorporated into intelligent agents. These agents monitor designers' actions and provide design guidance and assistance. The agents interact with objects in the environment and with designers. This paper describes the features of the proposed framework, design semantics and activities that agents are expected to support.
\end{abstract}

\section{Introduction}

Architectural representations and designs have been revolutionised by CAD (computer aided design) systems. Virtual environments and virtual reality seem to be the next logical step in the path laid down by CAD with the potential of more extensive impact particularly on the way architects design, visualise and present their designs to clients. In virtual design environments for architecture, designers will be active viewers and players in real time interaction [1]. This interaction is the generator of the representations of the virtual environments. Such environments will not only support the designer in modelling buildings at any level of complexity, but also support the development of conceptual design ideas of digital architecture [2].

Digital architecture is defined not just as a style, but as related to the major transformations and developments which globally affect our cities and places of social interaction [1]. Expanding bandwidth and computing power will increasingly affect our lives and will be manifested in the presence of digital artefacts in our physical environment. There are major changes being undertaken by the architectural profession not only at the design stage but also in its delivered artefacts. There seem to be possible shifts in the task of architecture by being digital including how the new media such as virtual environments will not only affect the design but also the built environment. This paper is not meant to provide answers to the latter part but rather looks at the former; that is how virtual environments can transform the design process and consultation and provide an appropriate design support to its users.

\section{Designing in virtual environments}

Current virtual environments allow users to construct their designs by providing them a platform within which they can compose their designs from various design elements, i.e. objects. In architectural design, architects are accustomed to investigating objects, spaces in their designs whereby objects play a dynamic role in building design. Current virtual environments, such as Active Worlds, provide users with the ability to build their own 3D models using objects that are available in the Active Worlds objects library or imported from CAD systems to augment the existing library. Building new objects with Active Worlds Browser is a three-step process. First users need to find some existing object to copy, or clone; that is the only way you can create a new object. This will be the starter object. Then, the object can be moved to the desired location. This object can be turned into an entirely different object by changing its object field in the object properties. Finally, the new object description and action can be changed. Each model is constructed from a set of objects, each object acts as a class of objects where its 
instances can be assigned different behaviours and attributes. However, direct manipulation of buildingobjects is extremely limited in current $3 \mathrm{D}$ virtual environments and inter-relationships between objects are not supported in most of the current virtual environments [3].

Designers tend to deal with spatial things in the way they perceive objects in $3 \mathrm{D}$ space and interact with the world. SCULPTOR [4] has been developed to demonstrate that conceptual designing is possible with the computer by offering real time manipulation of objects in space. SCULPTOR is equipped with certain features that can be used to generate and explore 3D models in a fluid and engaging environment. SCULPTOR as a design tool reflects the duality between space and materials. Room objects are simply a group of a larger solid with a smaller void inside. The difference is size defines the thickness of the wall. Moving rooms to intersect with each other automatically creates the more complex void shape of the inside with the possibility of hierarchical structures to build rooms inside rooms. SCULPTOR has these mechanisms that enable real-time modelling with intersecting materials, spaces and rooms. SCULPTOR provides the opportunity for collaborative modelling over the Internet which is controlled and managed on the web. On the collaboration management site users can start or join an existing SCULPTOR collaboration session. The actual state of the model can be observed through both pictures and VRML models that are uploaded and presented regularly.

Another application of Virtual Environments (VEs) in design and architecture includes the Conceptual Design Space CDS [5]. CDS is a real-time, interactive virtual environment application which attempts to address the issue of $3 \mathrm{D}$ design in general and immersive design in particular. Users of CDS can create simple conceptual building designs in an interactive, intuitive manner, simply by choosing vertices on the ground, then adding the third dimension by specifying a height for each vertex. The walls and ceiling are created automatically by CDS. Once the basic structure is in place, users may experiment with different colours, textures, add furniture to the interior of the space, or change the roof line, for example. Within CDS users will not only be able to inspect and inhabit their buildings, but will also have the ability to modify them, add details, or create new designs, all while immersed in the VE.

One would expect such VEs for architectural design to have knowledge about the designed entities. Navigating and manipulating in 3D requires not only 3D geometrical primitives, but also a set of 3D design tools. In order for a virtual environment to be effective as a designing platform for digital architecture, it is not enough to focus on the precision of the renderings and the accuracy of simulated behaviours. The environment should provide appropriate design support to its users. Design objects are rarely isolated; they are always in the company of other objects. Design spaces are constructed from the relationships between these objects. This paper presents a framework of developing virtual environments in which design semantics (high level relationships between objects) are incorporated into intelligent agents. The focus of the semantic-based agents in a virtual environment is to provide constructive informational and design-shaping feedback. The semantic-based framework proposed in this paper is distinguished by the intelligent response and feedback given to the designer during the initial design phase as well as at evaluation stages of designing.

\section{Design semantics in architecture}

Architects use a great variety of concepts while designing: geometrical shapes, spaces, building elements, user activities, structure, etc. Within this vocabulary, different design approaches are possible. Some designers follow a top-down while others proceed using a bottom-up approach. In both approaches, the early design ideas developed at conceptual stage of the design process have the biggest impact on the final design of the artefact. Each design idea encapsulates design knowledge that can be ascribed to the design artefacts. The design artefact is composed of sets of design objects or elements. Design semantics are exhibited in the high level relationships between these design elements or between spaces and shapes created using these elements.

Different building types have fundamentally different design rules and semantics, e.g. hospitals have one set and housing have another. Within these building types, different sets of semantics arise [6]. The design semantics include functional, spatial, esthetical, environmental and contextual semantics of building designs. In architectural designing, as in many other design disciplines, design composition is an important design activity wherein designers use building-objects to construct design compositions. Through design compositions designers express ideas and represent elements of design, abstract concepts and construct situations through the relationships between the parts of design compositions.

In architecture, spatial design is ubiquitous. Spatial design consists of the shapes that are used to delineate space, the spaces between the shapes, the relationships of the shapes and the spaces to each other, and the meaning or purpose attached to the spaces and their relationships. This meaning is what is often referred to as the semantics of architectural space. The semantics of space are derived from many sources and are manifested at many levels of abstraction. In the overall design of a building, the architect may intend to express some broad cultural sentiment. Typically, within this statement there will be several primary functions that require spatial expression. 
Other spaces may then be required to link and support the primary spaces [7].

This paper focuses on developing a framework that supports both spatial and esthetical semantics. Spatial semantics include segregation (privacy), interconnections (adjacency and links) where adjacency reflects the contiguity among elements in the design composition. Esthetical semantics include expression, symmetry and modality. Expression indicates the impression of a feature or a defined assemblage of features within the composition such as dominance. Visual dominance reflects the effect of an element size (e.g. a room or a space) and spatial location in relation to other elements. Symmetry indicates harmony and conformity among building elements within the composition such as reflective symmetry, cyclic rotation and translational repetition. Modality includes the characteristics of how these parts in the design composition are put together such as centrality, linearity and radiality [8].

\section{Intelligent agents in virtual environments}

The combination between virtual environments and intelligent agents has given rise to a new area at their meeting point, which is called intelligent virtual environments. The potential benefits of employing intelligent agents in virtual environments include empowering computers not only to support a much higher degree of visual realism but also with processes of intelligent behaviour. Research in the field of virtual environments is moving towards intelligent virtual environments in order to include specific options of functionality; e.g. researchers in the field of artificial life have even more ambitious aims. These aims include the creation of virtual worlds containing digital life with new rules but not necessarily similar to those of real world. Some see distributed interactive virtual environments such as Active Worlds as a basis for the development of such virtual worlds allowing autonomous interaction between artificial life forms and virtual worlds [9].

Agents interact with other agents and possibly humans via agent communication language. Intelligent agents exhibit the following attributes: autonomy, reactivity, proactivity and social ability. The autonomy of intelligent agents is reflected by the ability to learn without the direct intervention from human or other agents and having some kind of control over their interactions and internal state. For Intelligent agents to be reactive they should be able to perceive their environment and respond to changes that occur in it.

Engeli and Kurmann [10] view intelligent agents to keep the overview of the project, take over tedious tasks, or look up external information. Besides this, agents may influence the quality of human-computer collaboration. Engeli and Kurmann proposed five characteristics for the agent. The agent contains knowledge, it is designed to work on a specific task, it can work autonomously, it acts on behalf of the user, and it has the ability to learn. Engeli and Kurmann viewed two basic kinds of agents: design assisting agents and design generating agents. Design assisting agents help the user by providing information and executing background tasks. Design generating agents interfere with the design and come up with design solutions. Engeli and Kurmann have implemented three prototypical agents: Navigator, Sound Agent and Cost Agent. The three agents act as personal assistants trained by each user to adapt to one's individual preferences. The Navigator agent acts like a guide in the virtual world. It can follow different kind of instructions, e.g. moving to a specific place, moving in a specific direction or composing a tour. The Sound agent is a companion of the Navigator and tries to enhance the perception of the space by adding an auditory component to the visual impression. The cost agent estimates the cost of the project and displays the results graphically.

There are more potential advantages to support designing by introducing intelligent autonomous agents with design capabilities into the virtual environments. Such agents will not only offer designers advice about design semantics but actually move in the virtual environment, manipulate objects and invoke a set of operations to achieve a preferred design semantic nominated by the designer. Because the designer and the agent are in the same virtual environment, agents will have the opportunity to learn from designers' actions respond to them by making changes in the environment. The framework of proposed agents is introduced in this paper.

\section{Semantic-based design support in Virtual Environments}

When trying to identify primary design concepts in buildings, we see that all buildings invariably consist of floors, walls, roofs. The materials vary widely but the concept of enclosure remains constant. Every building incorporates structure and systems including plumbing, electrical, heating, and cooling and humidity control (HVAC), security, fire, networking, etc. In an anatomical sense, if the structure is the skeleton or skin, systems like heating and air conditioning are the organs of a building, contributing to its life and the inhabiting life forms. All of the above are considered building components and their primary purpose is to provide functional spaces for use. How spaces are arranged plays a subtle melody to our physical sensors. There are functional and objective aspects of designing spaces in architecture in addition to cultural, emotional and even ritual considerations. This is inherently linked to the perception of space. For instance, as we pass from exterior to interior, the subtle embrace of 
enclosure surrounds us. A museum may contain large central spaces with galleries attached or clearly defined. A grocery store is radically different. Multiple parallel aisles are arrayed with innumerable options for the user to browse, identify goods and purchase items. The functional needs, aesthetic preferences and are amongst the most important factors that influence design concepts. Design concepts are ideas identified by the designer as a foundation for composing a form to a space or a building [11].

Concepts are considered as knowledge or data abstracted from existing entities. Hence, the concept is the abstraction of real world from the procedure identifying and classifying parts on the basis of similar properties. So the structure of the concept is an application of the real world. Elements and relationships are contents of the concept as well as the real world. Architectural elements are columns, walls, spaces, etc. Relationships include spatial, contextual and esthetical relationships. These architectural elements can be further integrated and utilised to generate preliminary or sophisticated architectural designs concepts; an example is shown in Figure 1.
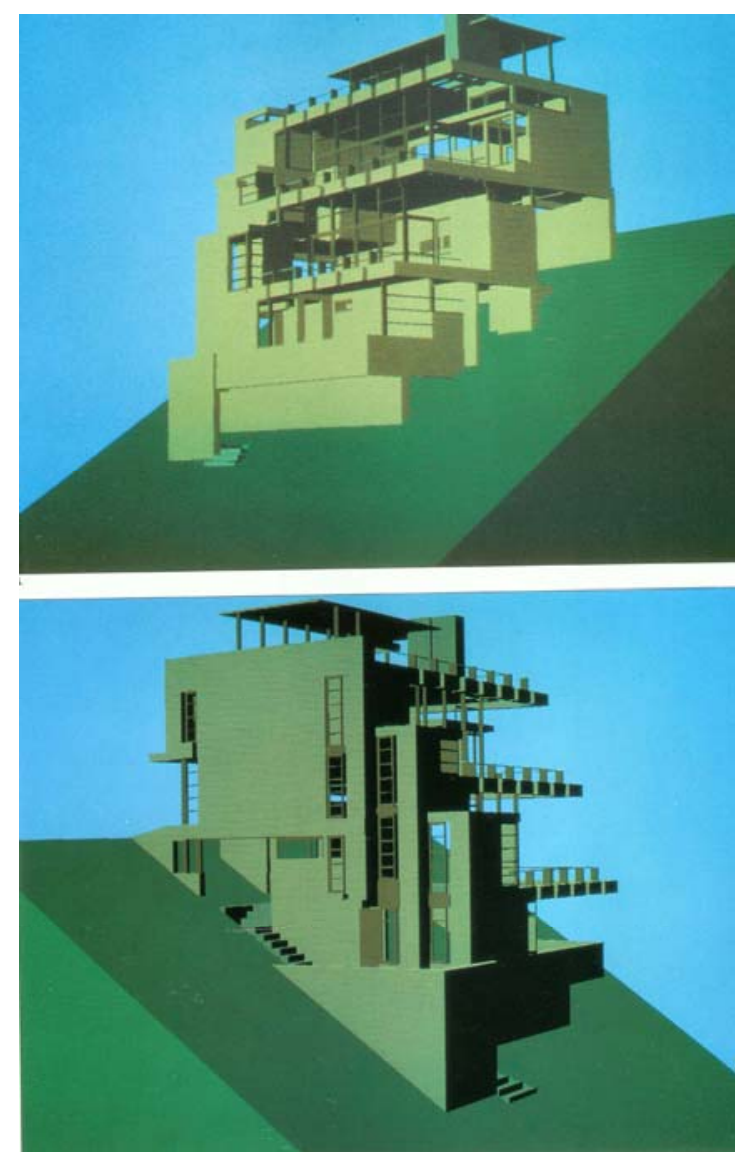

Figure 1. A design example composed of preliminary architectural elements. [12].

\section{A framework of sematic-based Agents to Support Designing in Virtual Environments}

\subsection{Structure of a situated action agent}

In order to successfully integrate intelligent autonomous agents in the context of virtual environments, a number of issues should be addressed. The framework should permit agent to use its knowledge to explain tasks and observe designers actions, as well as allowing the agents to perform actions at their discretion. In contrast, current agent frameworks focus on execution. They are not able to explain to the users what they are doing nor recognising what other agents or users are responding to [13]. The framework of intelligent agents should allow agents to act both reactively and in a goal directed manner. Hence, the agents' actions will be relevant both to the task at hand and to the current state of the environment. At the same time the agent must be able to perceive the virtual world as well as control its realisation in the virtual world with the ability to manipulate objects in the world.

In this research we are adapting the agent structure proposed by Russell and Norvig [14] where an agent can be viewed as perceiving its environment through sensors and acting upon that environment through effectors as depicted in Figure 2. The type of agent adapted here is a situated goal-based agent in which the agent has initial explicit goals but these goals can be further adapted by the agent depending on the internal state of the agent, agent's previous experience and the current state of the environment. Hence, such an agent is much richer than a simple reflex agent (condition-action rules) since the agent has a capacity to learn, modify what it learns and choose appropriate set of actions to the current situation. A proposed structure of the situated goal-based (action) agent is shown in Figure 3.

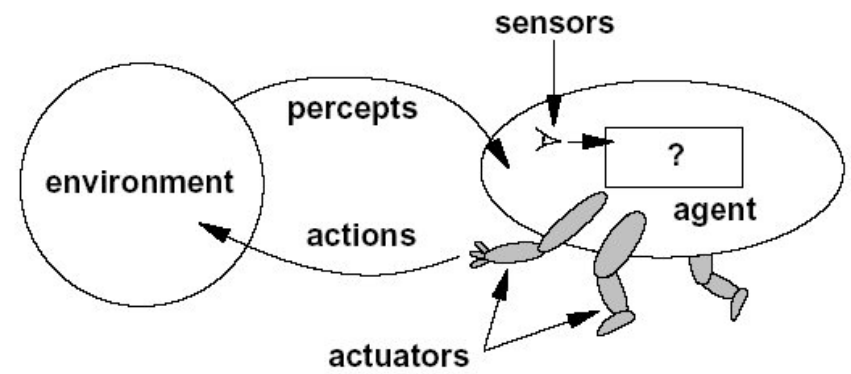

Figure 2. An Agent interacts with the environment through sensors and effectors [14].

The situated action approach to agents allows them to perform in different situations by changing their focus and situating themselves in the new situation. Such agents will exhibit situated behaviour. This is unique compared to 
specifying agent's behaviour by means of production rules. Encoding task behaviour as production rules denotes that the behaviour of such agents will be deterministic. The situated goal-based agent acquires its knowledge from: the situations it encounters, the environments it interacts with and designers' actions within these environments.

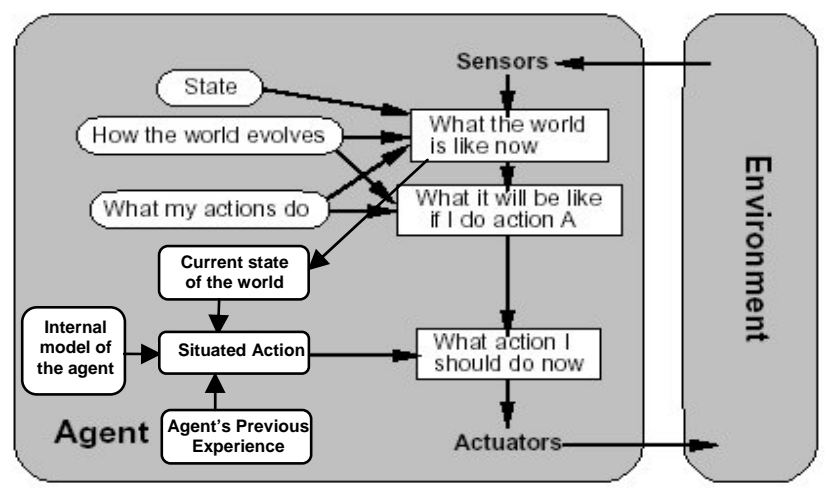

Figure 3. Proposed structure of a situated goal-based (action) agent.

\subsection{A framework of a multi agent system to support designing in a virtual environment}

The proposed framework of a multi agent system to support designing in virtual environments is organised in a two-tiered structure comprising design tools and design semantics support. The design tools tier focuses on enhancing the interface of virtual design environments by introducing adaptable design objects to facilitate the development of architectural designs. The adaptable design objects include walls, doors, windows, floors, slabs, roofs, stairs and furniture. These objects allow designers to modify their geometrical and non geometrical attributes. The adaptable design objects are not intelligent agents but rather intelligent objects. The intelligence of these objects is manifested in their capability to respond to agent's queries and follow agent's instructions. The intelligent abject are equipped with certain operations that allow them to move, rotate, cut, and scale when they are instructed by the situated action agent.

The design semantic support tier focuses on providing semantic support to human designers during the design process. The semantic support is primarily spatial and esthetical. Spatial semantics include segregation, interconnections and adjacency. Esthetical semantics include expression, symmetry and modality. The semantic support is provided by the situated action agent. The multi agent system is comprised of agents at three levels: Observation-Sensation-Perception, Conception and Action as shown in Figure 4.

Within the proposed framework, the Observer agent tracks designer's actions and their sequences including selecting and locating design objects and moving or deleting objects. The Sensor agent detects design objects whenever they are placed or modified in the virtual environment. The Preceptor agent builds its interpretation of the current design in the virtual environment through what has been sensed and observed. The Conceptor agent is an incremental learning agent that has pre-coded descriptions of spatial and esthetical semantics. The relationships between design semantics are not pre-coded but rather learned by the Conceptor agent. It learns from observing the interaction between the designer and the virtual environment and other agents including the Situated Action agent. The Situated Action agent decides its course of actions in response to designer's actions based on its previous experience and its internal model of the current design in the virtual environment. These actions are manifested either in changing the current design in the virtual environment or advising the designer in relation to a specific design semantic in his/her current design The proposed Situated Action agent does not follow a series of rules in response to pre-programmed set of design values but rather these values are learned by the agent in relation to specific and encountered situations. What separates it from the many similar agents that have been developed in recent years are its situatedness supported by its learning capability, its capability to modify its previous experiences in an incremental learning fashion and its situated capability to decide the best set of actions to be invoked in future situations.

\section{Discussion}

The integration of semantic-based intelligent agents and virtual environments has great potentials to support designers at the early stages of designing. The proposed framework of a multi agent system within virtual environments provides a platform for such integration. The interaction between agents and virtual environments, agents and the designer is essential to provide useful design support. Agents have different levels of interactions with the environment and the designer. These levels range from tracking designer's actions to introducing changes in the environment or advising designers of certain design semantics. The agent's capability to learn the relationships between design semantics and build its own design experience allows the agent to perform and act in future situations. The agent takes the best course of actions in future situations based on its previous knowledge aligned with the current situation in the environment.

The potential benefits of developing semantic-based intelligent design agents within virtual environments include improving the design support at the early stage of architectural design. The improvement is in terms of efficiency, satisfaction and effectiveness. Efficiency is the resources expended in relation to the accuracy and 
completeness with which designers achieve goals. Effectiveness is the accuracy of completeness with which designers achieve specific goals. Satisfaction is the freedom from discomfort and positive attitudes to the use of the design tool. Agent capabilities to track designer's actions, advise of recognised design semantics and introduce design changes in relation to designer's actions in real time will assist designers to achieve their goals without being constrained as using CAD tools. Since designers at the early design stage need freedom and absence of strictly defined rules

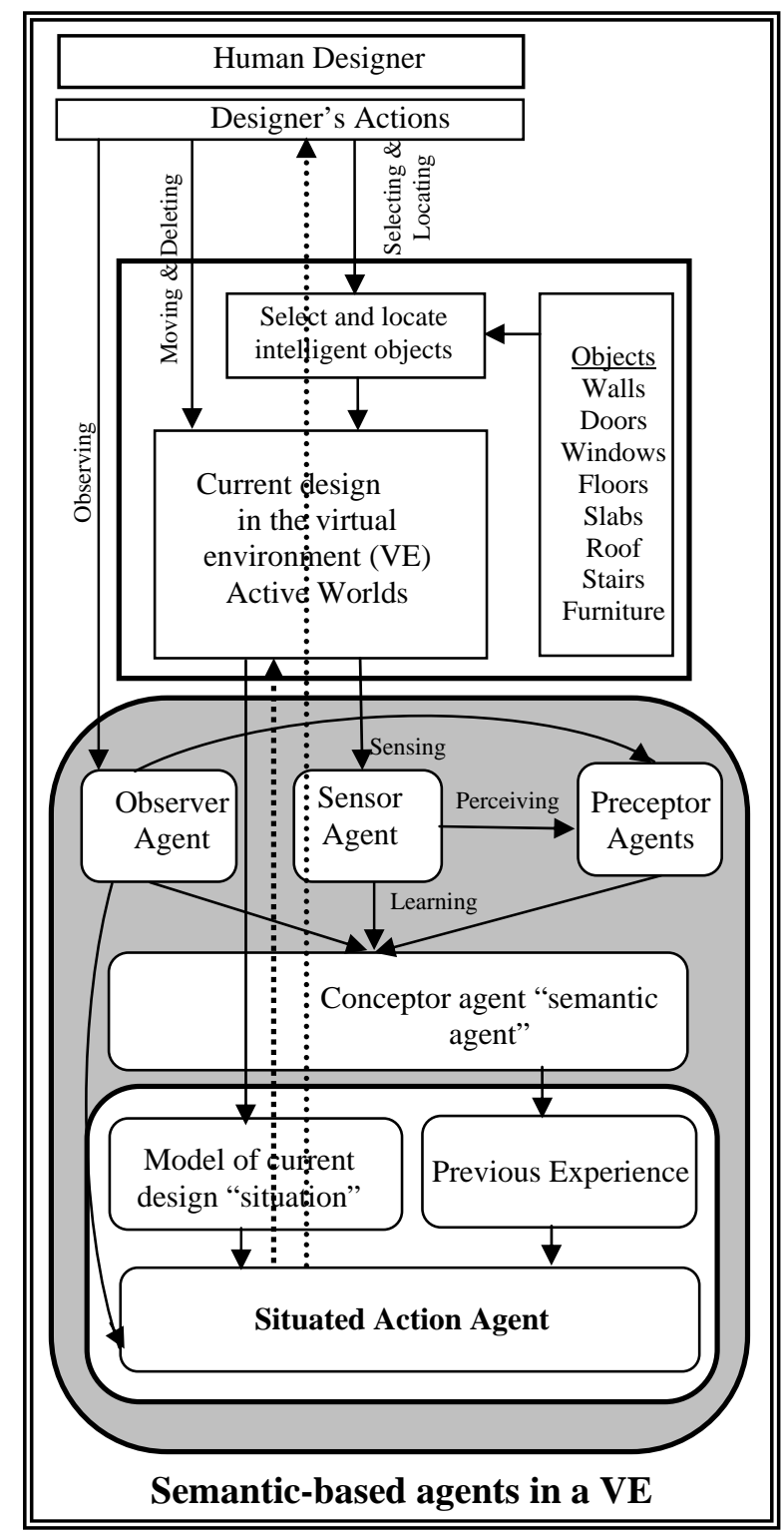

Figure 4. Proposed framework of a multi agent system to support semantic-based designing in virtual environments.

\section{References}

1. D. Bertol, Designing Digital Space: An Architect's Guide to Virtual Reality, John Wiley \& Sons, New York, 1997.

2. M. Engeli and D. Kurmann, Spatial objects and intelligent agents in a virtual environment, Automation in Construction, 1996, 5: pp. 141-150.

3. R. Reffat, Semantic-Based Virtual Design Environments for Architecture, Proceedings of Education of Computer Aided Architectural Design in Europe (eCAADe), 2003, to appear.

4. D. Kurmann, SCULPTOR - How to design space, in $\mathrm{T}$. Sasada, S. Yamaguchi, M. Morozumi, A. Kaga and R. Homma, Proceedings of the Third Conference on Computer Aided Architectural Design Research in Asia, Osaka University, Osaka, Japan, 1998, pp. 317325.

5. D. Bowman, Conceptual Design Space: Beyond Walkthrough to Immersive Design, in D., Bertol, (ed.) Designing Digital Space, John Wiley \& Sons, New York, 1997, pp. 225-236.

6. C. Eastman, A data model for design knowledge, in G. Carrara and Y. Kalay (eds), Knowledge-Based Computer-Aided Architectural Design, Elsevier Science, Amsterdam, 1994, pp. 95-122

7. P. G. McIntosh, Models of spatial information in computer-aided architectural design: a comparative study, in Y. E. Kalay (ed), Computability of Design, John Wiley \& Sons, New York, 1987, pp. 117-140.

8. R. Reffat and J. Gero, Computational situated learning in design, in Gero, J. S. (ed.), Artificial Intelligence in Design'00, Kluwer, Dordrecht, 2000, pp. 589-610.

9. R. Aylett and M. Luck, Applying artificial intelligence to virtual reality: intelligent virtual environments, Applied Artificial Intelligence, Vol. 14 (1), 2000, pp. 3-32.

10. M. Engeli and D. Kurmann, Spatial objects and intelligent agents in a virtual environment, Automation in Construction, vol 5, 196, pp. 141-150.

11. S. Miller, Design Process: A Primer to Architectural and Interior Design, Van Nostrand Reinhold, New York, 1995.

12. L. Mrach and J. Sheine, RM Schindler: Composition and construction, Academy Editions, London, 1993.

13. W. L. Johnson, J. Rickel, R. Stiles and Munro Allen, Integrating pedagogical agents into virtual environments, Presence: Teleoperators and Virtual Environments, 1998, vol 7(6): pp. 523-546.

14. S. Russel and P. Norvig, Artificial Intelligence: A modern Approach, Prentice-Hall, Upper Saddle River, NJ, 1995. 